\title{
Magnetic Domains and Stripes in the Spin-Fermion Model for Cuprates
}

\author{
Charles Buhler, Seiji Yunoki and Adriana Moreo \\ Department of Physics, National High Magnetic Field Lab and MARTECH, \\ Florida State University, Tallahassee, FL 32306, USA
}

(August 11, 2018)

\begin{abstract}
Monte Carlo simulations applied to the Spin-Fermion model for cuprates show the existence of antiferromagnetic spin domains and charge stripes upon doping. The stripes are partially filled, with a filling of approximately $1 / 2$ hole per site, and they separate spin domains with a $\pi$ phase shift among them. The stripes observed run either along the $\mathrm{x}$ or $\mathrm{y}$ axes and they are separated by a large energy barrier. No special boundary conditions or external fields are needed to stabilize these structures at low temperatures. When magnetic incommensurate peaks are observed at momentum $\pi(1,1-\delta)$ and symmetrical points, charge incommensurate peaks appear at $(0,2 \delta)$ and symmetrical points, as experimentally observed. The strong charge fluctuations responsible for the formation of the stripes also induce a pseudogap in the density of states.
\end{abstract}

PACS numbers: $74.20 . \mathrm{Mn}, 71.10 . \mathrm{Fd}, 74.25 . \mathrm{Ha}$

In recent years neutron scattering experiments have established that magnetic incommensurability is a property common to most of the high-Tc cuprates [1]. In addition, there is mounting evidence supporting charge stripe formation in these compounds as well. [2] These nontrivial spin and charge arrangements may be crucial to understand the unusual transport and superconducting behavior of the cuprates. Early Hartree-Fock studies of the Hubbard model already predicted stripe formation with insulating characteristics. [3] Phase-separation between hole-rich and hole-poor regions in the $\mathrm{CuO}_{2}$ planes, supplemented by long-range Coulomb interactions, has also been proposed to explain the existence of stripes. [4] In addition, ground states with metallic stripes made out of $\mathrm{d}$-wave hole pairs have been observed in the $\mathrm{t}-\mathrm{J}$ model using special boundary conditions. [5] However, a more detailed theoretical understanding of these phenomena and resolution of current conflicting results have been extremely challenging especially since the t-J and Hubbard models used for the cuprates are considerably difficult to study. As an alternative to this more traditional approach here we present the first computational study of a simpler phenomenological model, the Spin-Fermion (SF) model, which has been previously analyzed mostly using mean field approximations with the main goal of understanding d-wave superconductivity. [6] B] Here the focus is instead shifted toward the magnetic and charge properties of the SF model, which have not been explored before using unbiased techniques. In carrying out such a study unexpected results were observed, notably the presence of spin incommensurability and metallic stripe formation at finite hole density, in excellent agreement with experiments.

The SF model is constructed as an interacting system of electrons and spins, mimicking phenomenologically the coexistence of charge and spin degrees of freedom in the cuprates. 6 8]. Its Hamiltonian is given by

$$
\mathrm{H}=-\mathrm{t} \sum_{\langle\mathbf{i}\rangle \alpha}\left(\mathrm{c}_{\mathbf{i} \alpha}^{\dagger} c_{\mathbf{j} \alpha}+\text { h.c. }\right)+\mathrm{J} \sum_{\mathbf{i}} \mathbf{s}_{\mathbf{i}} \cdot \mathbf{S}_{\mathbf{i}}+\mathrm{J}^{\prime} \sum_{\langle\mathbf{i} \mathbf{j}\rangle} \mathbf{S}_{\mathbf{i}} \cdot \mathbf{S}_{\mathbf{j}},
$$

where $\mathrm{c}_{\mathbf{i} \alpha}^{\dagger}$ creates an electron at site $\mathbf{i}=\left(i_{x}, i_{y}\right)$ with spin projection $\alpha, \mathbf{s}_{\mathbf{i}}=\sum_{\alpha \beta} \mathrm{c}_{\mathbf{i} \alpha}^{\dagger} \sigma_{\alpha \beta} \mathrm{c}_{\mathbf{i} \beta}$ is the spin of the mobile electron, the Pauli matrices are denoted by $\sigma, \mathbf{S}_{\mathbf{i}}$ is the localized spin at site $\mathbf{i},\langle\mathbf{i j}\rangle$ denotes nearest-neighbor (NN) lattice sites, $t$ is the NN-hopping amplitude for the electrons, $\mathrm{J}>0$ is an antiferromagnetic (AF) coupling between the spins of the mobile and localized degrees of freedom (DOF), and $\mathrm{J}^{\prime}>0$ is a direct AF coupling between the localized spins. The density $\langle n\rangle=1-\mathrm{x}$ of itinerant electrons is controlled by a chemical potential $\mu$. Hereafter $t=1$ will be used as the unit of energy. From previous phenomenological analysis the coupling $\mathrm{J}$ is expected to be larger than $\mathrm{t}$, while the Heisenberg coupling $\mathrm{J}^{\prime}$ is expected to be smaller. [7, 8]

Here classical spins with $\left|S_{\mathbf{i}}\right|=1$ will be used for the localized spins, as also assumed in previous literature. 99 This will allow us to perform Monte Carlo (MC) simulations of model Eq.(1) without "sign problems", reaching by this procedure temperatures as low as $\mathrm{T}=0.01$ at any density. This temperature is well below $\mathrm{T}=0.2$, the lowest that can be stabilized with quantum MC away from half-filling in the standard Hubbard model. [10] The value of J will be fixed to 2, as suggested in Ref. 8] where comparisons with experimental results were performed. The coupling $\mathrm{J}^{\prime}$ among the classical spins will be set to 0.05 . This value was selected by monitoring the magnetic susceptibility and comparing its behavior to experimental results for the cuprates. The present study has been performed mostly on $8 \times 8$ lattices with periodic boundary conditions (PBC), but occasional runs were made also using open and antiperiodic BC as well as different lattice sizes. The numerical technique used here involves a 
standard Metropolis algorithm for the classical spins and an exact diagonalization for the itinerant electrons. The details of the method have been described in Ref. [11].

To study the magnetic properties of the system we measured the spin-spin correlation functions among the classical spins defined as $\omega(\mathbf{r})=\frac{1}{N} \sum_{\mathbf{i}}\left\langle\mathbf{S}_{\mathbf{i}} \cdot \mathbf{S}_{\mathbf{i}+\mathbf{r}}\right\rangle$, where $\mathrm{N}$ is the number of sites. The Fourier transform of $\omega(\mathbf{r})$, i.e. the spin structure factor $S(\mathbf{q})$, was also investigated. The momentum $\mathrm{q}_{\gamma}$ takes the values $2 \pi \mathrm{n} / \mathrm{L}_{\gamma}$, with $\mathrm{n}$ running from 0 to $\mathrm{L}_{\gamma}-1$, and $\mathrm{L}_{\gamma}$ denoting the number of sites along the $\gamma=\mathrm{x}$ or $\mathrm{y}$ direction. In our MC simulations long-range AF order has been observed at $\langle n\rangle=1.0$ as expected. As the electron density is reduced from 1 , the $S(\pi, \pi)$ intensity decreases. One of the main results observed in this effort occurs at finite hole density where a remarkable spin incommensurability appears at $\langle\mathrm{n}\rangle \approx 0.8$ with the structure factor peak moving to $\pi((1-\delta), 1)=(3 \pi / 4, \pi)$ and rotated points. This behavior is illustrated in Fig.1-a where $S(\mathbf{q})$ on $8 \times 8$ lattices at $\mathrm{T}=0.01$ is shown for different values of $\langle\mathrm{n}\rangle$ along the path $(0,0)-(0, \pi)-(\pi, \pi)-(0,0)$. Results along $(0,0)-(\pi, 0)-(\pi, \pi)-(0,0)$ are in the inset.
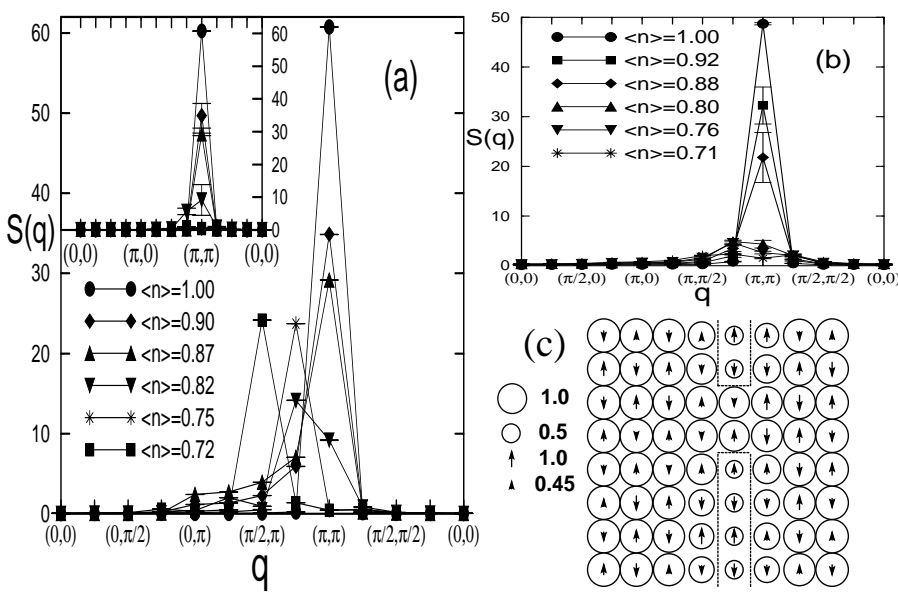

FIG. 1. (a) Structure factor $S(\mathbf{q})$ for the localized spins versus momentum for $\mathrm{J}=2, \mathrm{~J}^{\prime}=0.05$ and $\mathrm{T}=0.01$ on an $8 \times 8$ lattice and several densities along $(0,0)-(0, \pi)-(\pi, \pi)-(0,0)$ (inset: results along $(0,0)-(\pi, 0)-(\pi, \pi)-(0,0))$ ); (b) same as (a) but at $\mathrm{T}=0.05$; (c) spin and charge distribution for a typical MC snapshot at $\langle\mathrm{n}\rangle=0.9$ and the same parameters as in (a). The arrow lengths are proportional to the local spin $S_{z}(\mathbf{i})$ and the radius of the circles is proportional to the local density $n(\mathbf{i})$, according to the scale shown. PBC are used.

Note that the spin correlations along the two paths of Fig.1-a are different because at low temperatures the symmetry under lattice $\pi / 2$-rotations appears spontaneously broken. Similar ground-state properties but rotated by $\pi / 2$ have also been observed in independent runs depending on the initial conditions 12 for the classical spins indicating that there are two energy minima separated by a large barrier. At $\mathrm{T}=0.05$ (Fig.1-b) and higher temperatures the rotational symmetry is restored but the intensity of the incommensurate (IC) peaks is considerably reduced (e.g. at $\langle n\rangle=0.75$ the $\mathrm{T}=0.01 \mathrm{IC}$ peaks are four times higher than at $\mathrm{T}=0.05)$. This may explain the low intensity of the IC peaks observed in the $\mathrm{T}=0.2$ Hubbard model quantum MC simulations. [10]

Experimentally it was observed that $\delta \approx 0.25$ corresponds to the saturation value reached at hole density $\mathrm{x} \approx 0.12$, which persists up to $\mathrm{x} \approx 0.25$. [13] Due to the finite size of the lattices studied here, incommensurability for values of $\delta$ smaller than 0.25 cannot be detected apparently preventing us from comparing directly with the low-doping experimental results. However, we have analyzed typical spin configurations (snapshots) emerging from our MC simulations close to $\langle n\rangle=1.0$ and we observed the existence of large AF spin domains in most of them, an example of which is shown in Fig.1-c for $\langle\mathrm{n}\rangle=0.9$. The existence of these domains clearly suggests that tendencies towards magnetic incommensurability appear in the system at $\mathrm{x} \leq 0.20$ as well.

The origin of the short-range incommensurate magnetic order in the cuprates is still not clear. Magnetic order due to charge order has been proposed as a possible explanation. To explore the possibility of charge ordering in the SF model, $N(\mathbf{q})$ was studied here defined as the Fourier transform of the charge correlations $\mathrm{n}(\mathbf{r})=\frac{1}{\mathrm{~N}} \sum_{\mathbf{i}}\left\langle\left(\mathrm{n}_{\mathbf{i}}-\langle\mathrm{n}\rangle\right)\left(\mathrm{n}_{\mathbf{i}+\mathbf{r}}-\langle\mathrm{n}\rangle\right)\right\rangle$, where $\mathrm{n}_{\mathbf{i}}$ is the number operator at site $\mathbf{i}$ for the itinerant fermions. In the AF phase at $\langle\mathrm{n}\rangle=1.0 N(\mathbf{q})$ was observed to present a broad peak at $\mathbf{q}=(\pi, \pi)$ due to negative charge correlations (charge repulsion) at very short distances, in agreement with previous calculations [14]. As the system is hole doped the behavior of $N(\mathbf{q})$ becomes more temperature dependent. At $\mathrm{T}=0.05$ the peak in $N(\mathbf{q})$ remains at $(\pi, \pi)$, but at $\mathrm{T}=0.01$ a sharp peak appears at small momenta for $0.7<\langle\mathrm{n}\rangle<0.9$ (Figs.2-a,b) indicating the existence of extended charge structures. If charge and spin incommensurability were related, stripe studies [4] predict that the peak in $N(\mathbf{q})$ has to appear at $(2 \delta, 0)$ and symmetrical points. Then, incommensurability associated to $\delta=0.125$ that cannot be explicitly detected in $S(\mathbf{q})$ due to the size of our clusters can nevertheless be observed in the charge channel. Indeed in Fig.2-a the peak in $N(\mathbf{q})$ for density 0.87 is located at $\mathbf{q}=(\pi / 4,0)$ compatible with $\delta \approx 0.125$. At low temperature the peak can be observed along the $\mathrm{x}$ direction but not along y (see inset of Fig.2-a) indicating that in the $\mathrm{MC}$ runs described here the charge domains are along the y direction, causing the spontaneous breaking of rotational symmetry described before. This clearly can be seen in the charge distribution MC snapshot at $\langle n\rangle \approx 0.85$ shown in Fig.3-a. Note that the holes are located along the magnetic domain boundaries. The presence of stripes was explicitly verified also using $12 \times 12$ clusters. The result is in excellent agreement with experiments [15] on Nd-doped LSCO where vertical stripes are observed at the Sr concentration $\mathrm{x} \approx 1 / 8$.

In some stripe scenarios 16 charge ordering is expected to occur at higher temperature than magnetic or- 
der. However, in model Eq.(1) both charge and magnetic ordering appear to occur at similar temperatures. Consider, for example, in Fig.3-b a typical MC snapshot at $\langle\mathrm{n}\rangle=0.75$ and $\mathrm{T}=0.01$. The holes are here aligned along two vertical columns. $S(\mathbf{q})$ and $N(\mathbf{q})$ for this particular snapshot are very similar to the averages shown in Fig.1-a and 2-a, clearly indicating incommensurate behavior in spin and charge. As the temperature is raised to $\mathrm{T}=0.05$, stripes are no longer observed (Fig.3-c) but there are still hole-poor magnetic domains which produce the (small) incommensurate peak shown in Fig.1-b at this density. An equally weak feature appears in $N(\mathbf{q})$ but it is more difficult to distinguish because the background raises with increasing momentum, rather than being flat (Fig.2-b). This may be the reason why the spin incommensurability is easier to detect than charge inhomogeneities in models such as Hubbard or t-J, where low temperatures are difficult to reach. As it can be observed in the snapshot of Fig.3-c even at $\mathrm{T}=0.05$ hole-rich patches and antiferromagnetically spin aligned hole-poor domains coexist, suggesting that incommensurate charge and spin order occur simultaneously due to their mutual interactions in the SF model.
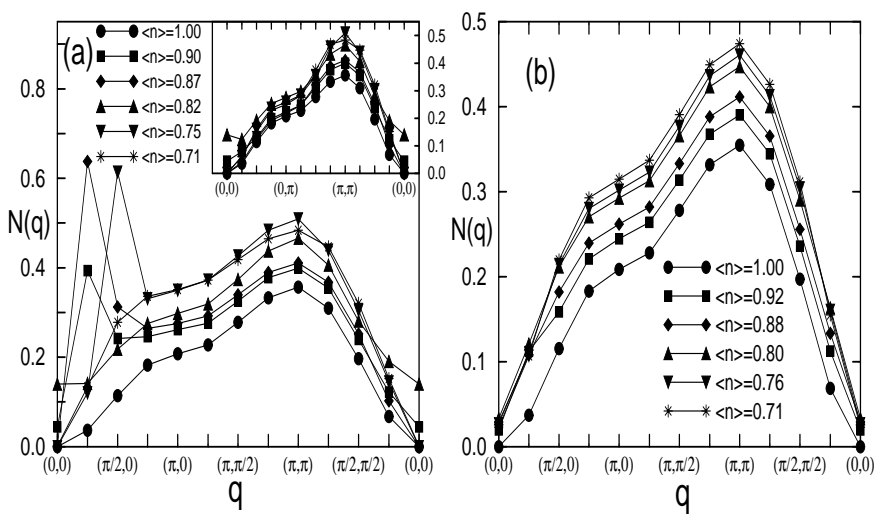

FIG. 2. (a) Structure factor $N(\mathbf{q})$ for the electrons versus momentum for $\mathrm{J}=2, \mathrm{~J}^{\prime}=0.05$ and $\mathrm{T}=0.01$ on an $8 \times 8$ lattice at several densities along the path $(0,0)-(\pi, 0)-(\pi, \pi)-(0,0)$ (inset: same as main figure but along $(0,0)-(0, \pi)-(\pi, \pi)-(0,0))$; (b) same as (a) but at $\mathrm{T}=0.05$. PBC are used.

Our results are in qualitative agreement with the conclusions of Ref. [5, 17] where it was argued that stripes can be stabilized at realistic values of $\mathrm{J} / \mathrm{t}$ in the $\mathrm{t}-\mathrm{J}$ model without the use of long-range Coulomb interactions. Moreover, in the SF model here we showed that charge stripes can appear spontaneously without the need of using external staggered magnetic fields or special BC to pin them. 17] The SF model provides a clean and easy to study framework for the analysis of stripe formation in models of correlated electrons. The origin of the stripes in our study can be understood in part by analyzing the behavior of $\langle\mathrm{n}\rangle$ vs $\mu$ shown in Fig.4-a, where it is observed that the density changes rapidly between 0.5 and 1 . At $\langle\mathrm{n}\rangle \approx 0.5$ there is a plateau indicating that this density is particularly stable. For $0.5 \leq\langle\mathrm{n}\rangle \leq 1$ substantial charge fluctuations are to be expected due to the large value of $d\langle\mathrm{n}\rangle / d \mu$, involving regions whith density close to 1 (spin domains) and to 0.5 (hole stripes) which correspond to the two highly stable densities that appear in the system. In fact, calculating the charge density along the stripes we found that $0.5 \leq\langle\mathrm{n}\rangle_{\text {stripe }} \leq 0.65$, which seems to indicate the existence of approximately one hole every two $\mathrm{Cu}$ ions. The charge density on the hole poor regions, on the other hand, has a value very close to 0.9 . These densities inside and outside the stripe are in excellent agreement with experiments [2]. The SF model improves on early Hartree-Fock calculations that predicted a stripe density close to zero.

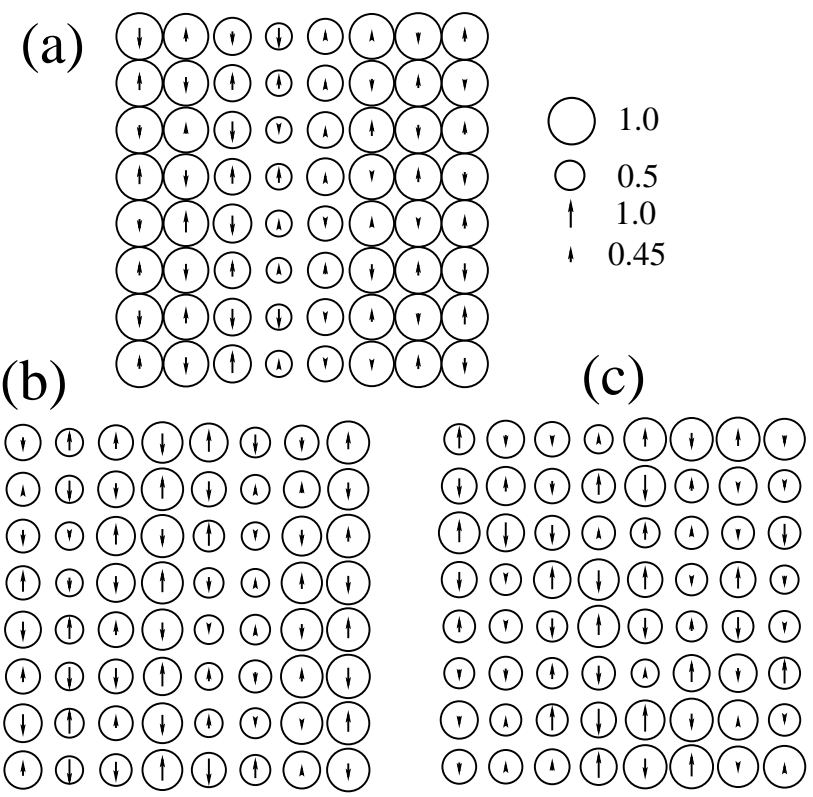

FIG. 3. (a) Spin and charge distribution for a MC snapshot on an $8 \times 8$ cluster for $\mathrm{J}=2, \mathrm{~J}^{\prime}=0.05, \mathrm{~T}=0.01$, and at $\langle\mathrm{n}\rangle \approx 0.85 ;(\mathrm{b})$ spin and charge distribution for a snapshot at $\langle\mathrm{n}\rangle=0.75$ and the same parameters as in (a). The notation is as in Fig.1-c; (c) same as (b) but for $\mathrm{T}=0.05$. PBC are used.

It is important to note that the SF model does not phase separate in spite of its large compressibility. Using different lattice sizes and $\mathrm{BC}$ we have verified that there is no discontinuity in $\langle\mathrm{n}\rangle$ vs. $\mu$, while the energy presents a nearly straight line behavior in the density range between 0.5 and 1.0 (Fig.4-b). Using starting MC configurations in which all the holes were together at the center of the cluster it was observed that this arrangement decays into stripes, and comparing the energy of the phase separated and stripe configurations the latter was found to have a lower energy than the former.

As remarked before, stripe configurations were observed with several BC including periodic. One subtlety encountered in the latter is that for densities where a single stripe is stabilized, the PBC prevented the occurrence of a $\pi$-shift in the spin domains because it would induce spin frustration (Figs.3-a). However, if PBC are replaced 
by open $\mathrm{BC}(\mathrm{OBC})$ the stripe still appears and in this case a $\pi$-shift is observed (Fig.4-c). When the number of stripes is even, as in Fig.3-b, the $\pi$-shift is spontaneously obtained independently of the $\mathrm{BC}$. This result is also in excellent agreement with experiments. 15.

Our simulations can produce dynamical information directly in real-frequency without the need of carrying out (uncontrolled) analytic extrapolations from the imaginary axis. This is particularly important to compare theoretical predictions with the results of recent photoemission experiments on optimally doped LSCO which showed the development of a pseudogap at $\mu$ as the temperature decreases. 18] In Fig.4-d we present the density of states (DOS) for $\langle\mathrm{n}\rangle=0.75$ at $\mathrm{T}=0.05,0.02$ and 0.01 . A pseudogap at $\omega=\mu$ clearly develops for decreasing temperatures. This is a consequence of the strong density fluctuations discussed above, and it is similar to the phenomenon recently observed in the context of manganites where a pseudogap develops due to the coexistence of hole-rich and hole-poor domains. [19]
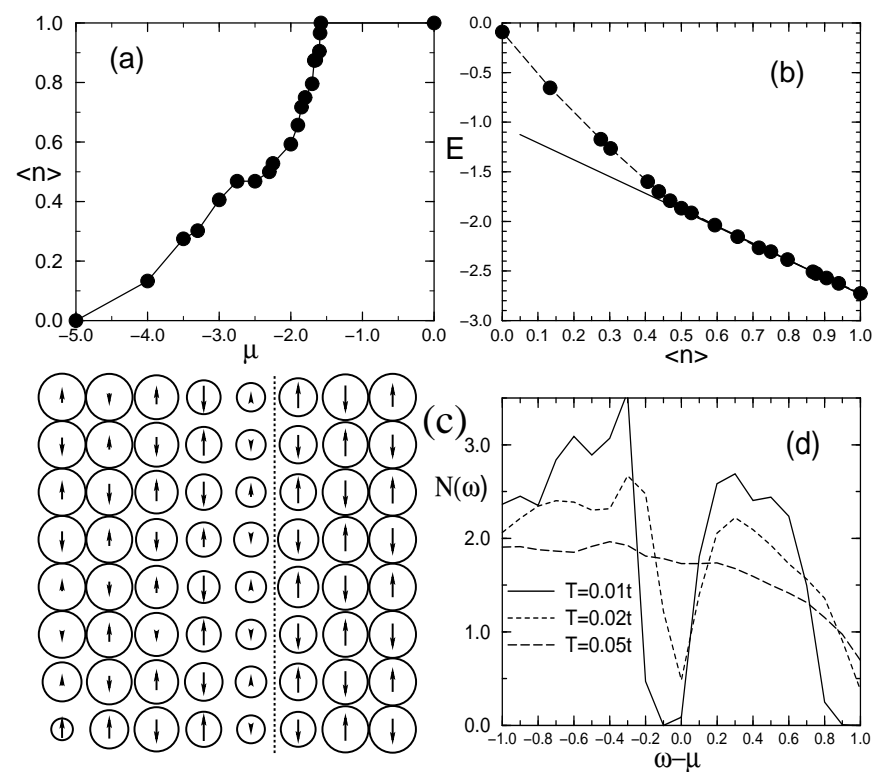

FIG. 4. (a) Density $\langle\mathrm{n}\rangle$ vs $\mu$ for $\mathrm{J}=2, \mathrm{~J}^{\prime}=0.05$ and $\mathrm{T}=0.01$ on an $8 \times 8$ lattice with PBC; (b) energy versus density for the same parameters as in (a); (c) spin and charge distribution for a $\mathrm{MC}$ snapshot at $\langle\mathrm{n}\rangle \approx 0.85$ and the same parameters as in (a) using OBC. The notation is as in Fig.3; (d) density of states as a function of $\omega-\mu$ at $\langle\mathrm{n}\rangle=0.75$ for different temperatures. The remaining parameters are as in (a).

Summarizing, the SF model has been studied using MC techniques without a-priori assumptions on their properties. Magnetic and charge incommensurability has been observed upon hole doping in this model. The incommensurability is due to the formation of AF domains separated by metallic stripes of holes. A $\pi$-shift is observed between the domains, and the stripes are partially filled with an electronic density of about 0.5. Charge and spin incommensurability appear correlated, and stripe- like configurations are obtained independently of the $\mathrm{BC}$ used and without the long-range Coulomb repulsion. The effect arises from the strong charge fluctuations between densities 0.5 and 1.0, which in addition produces a clear pseudogap in the DOS. Phase separation has not been observed in this study. The complex behavior of the SF model reported here, not anticipated in previous analysis, suggests that this model can be as useful for theoretical studies of the cuprates as the Hubbard and t-J models, while computationally it is considerably simpler.

A.M. is supported by NSF under grant DMR-9814350. Additional support is provided by the National High Magnetic Field Lab and MARTECH.

[1] S-W. Cheong et al., Phys. Rev. Lett. 67, 1791 (1991); P. Dai et al., Phys. Rev. Lett. 80, 1738 (1998); H.A. Mook et al., Nature 395, 580 (1998).

[2] J.M. Tranquada et al., Phys. Rev. Lett. 78, 338 (1997).

[3] D. Poilblanc and T. M. Rice, Phys. Rev. B39, 9749 (1989); J. Zaanen and O. Gunnarsson, Phys. Rev. B40, 7391 (1989).

[4] V.J. Emery and S.A. Kivelson, Physica C209, 597 (1993); ibid C235 189 (1994); and references therein.

[5] S. R. White and D. J. Scalapino, Phys. Rev. Lett. 80, 1272 (1998).

[6] P. Monthoux and D. Pines, Phys. Rev. B47, 6069 (1993); A. Chubukov, Phys. Rev. B52, R3840 (1995).

[7] J.R. Schrieffer, J. of Low Temp. Phys. 99, 397 (1995); B.L. Altshuler et al. Phys. Rev. B52, 5563 (1995).

[8] C.-X. Chen et al. Phys. Rev. B43, 3771 (1991).

[9] This is an approximation commonly used in the study of the SF model. See, for example, Ref. [6. The agreement with experiments found in our study provides an extra (a-posteriori) justification for this approximation.

[10] A. Moreo et al., Phys. Rev. B41 2313 (1990); D. Duffy and A. Moreo, Phys. Rev. B52 15607 (1995).

[11] S. Yunoki et al., Phys. Rev. Lett. 80, 845 (1998): E. Dagotto et al., Phys. Rev. B58, 6414 (1998).

[12] The initial conditions when not otherwise specified are random disordered spin configurations.

[13] K. Yamada et al., Phys. Rev. B57, 6165 (1998).

[14] Y.C. Chen et al., Phys. Rev. B50, 655 (1994).

[15] J.M. Tranquada et al., Nature. 375, 561 (1995).

[16] O. Zachar et al., Phys. Rev. B57, 1422 (1998).

[17] R. Eder et al., Phys. Rev. B60, R3716 (1999); S. White and D. Scalapino, preprint, cond-mat/9812187.

[18] T. Sato et al., preprint, to appear in Phys. Rev. Lett.

[19] A. Moreo et al., to appear in Phys. Rev. Lett. 